Les Cahiers du Gres

\title{
Action positive et égalité : une analyse sociologique
}

\section{Linda Pietrantonio}

Volume 2, numéro 1, automne 2001

URI : https://id.erudit.org/iderudit/009423ar

DOI : https://doi.org/10.7202/009423ar

Aller au sommaire du numéro

Éditeur(s)

Groupe de recherche ethnicité et société

CEETUM

ISSN

1499-0431 (imprimé)

1499-044X (numérique)

Découvrir la revue

Citer cet article

Pietrantonio, L. (2001). Action positive et égalité : une analyse sociologique. Les Cahiers du Gres, 2(1), 55-71. https://doi.org/10.7202/009423ar d'utilisation que vous pouvez consulter en ligne.

https://apropos.erudit.org/fr/usagers/politique-dutilisation/ 
Linda PIETRANTONIO, Ph.D

Département de sociologie

Université de Montréal

Action POSITIVE ET ÉGALITÉ :

UNE ANALYSE SOCIOLOGIQUE

Depuis trente ans, opinions et études scientifiques sur les politiques sociales dites d'action positive ${ }^{1}$ s'appuient sur la notion d'égalité pour favoriser la mise en place et la pratique de ces politiques, mais aussi pour les dénoncer. Le recours à la notion d'égalité dans l'argumentation sociale et scientifique sur l'action positive paraît obligé, cependant peu d'études se sont interrogées sur ces usages apparemment différenciés de l'égalité où celle-ci sert à la fois une argumentation <pro> et une argumentation $<$ contre $>$ ces politiques. Doit-on alors parler de l'ambiguïté du concept d'égalité ? À l'instar de Michel Pêcheux (1979), nous sommes d'avis que tout terme social d'usage important et qualifié d'ambigu révèle en fait la présence de rapports de force. Une étude de la structuration sociologique de la notion égalité à partir des catégories d'analyse $<$ majoritaire $>$ et <minoritaire $>$ nous a permis d'examiner sous un nouveau jour les débats de légitimation/délégitimation de l'action positive (Pietrantonio, 1999). Cette étude relève divers usages explicites et implicites de l'égalité et on peut lire à travers ces usages les rapports sociaux concrets entre groupes majoritaires et minoritaires, dans leur dimension discursive. $\mathrm{Ce}$ texte rend compte de cette analyse sociologique de l'égalité développée dans le cadre de l'examen du discours social scientifique sur l'action positive. L'étude démontre que l'ambiguiité du concept d'égalité au sein du discours social sur l'action positive renvoie en fait à des rapports sociaux de pouvoir.

\section{L'action positive ou de l'éga- lité juridique à l'égalité fac- tuelle}

Même s'il existe des différences notables quant aux types de mesures qui caractérisent la pratique de l'action positive selon les sociétés occidentales, ces politiques et leur conceptualisation sont en quelque sorte l'admission que l'égalité est un fait de structure. Mais les politiques d'action positive sont aussi liées à l'admission sociale que l'inégalité est aussi un fait de structure.

Ces mesures antidiscriminatoires partent du même substrat légal, et on y identifie, de la Suède au Québec en passant par les ÉtatsUnis, les mêmes constats et finalités : soit la persistance de pratiques discriminatoires (mises en évidence notamment sur le marché de l'emploi) à l'égard des femmes et autres groupes sociaux minoritaires et la mise en œuvre de moyens ou d'outils pour contrer ces pratiques qui passent par une spécification 
juridique de l'égalité.

Suivant la reconnaissance sociale du phénomène de discrimination systémique $^{2}$ et son ancrage juridique au sein des «droits à l'égalité », la plupart des législations sur l'action positive se caractérisent par trois dimensions (Delphy 1995) :

1. Au-delà de l'obligation législative que suggère le droit à l'égalité associé à la reconnaissance des droits de la personne ou des droits humains, les sociétés concernées par les mesures d'action positive doivent trouver les moyens de « mettre en œuvre » l'égalité. II n'y a toutefois pas d'obligation de conformité des mesures d'une société à l'autre, d'où la variété des mesures ${ }^{3}$.

2. À l'obligation de fonder des moyens de " mise en œuvre de l'égalité » s'ajoute l'obligation des " résultats "; mieux connue sous le nom de « égalité des résultats ". $\mathrm{Au}$ Canada, ces mesures sont perçues comme un préalable à l'exercice du droit à « l'égalité des chances » pour les groupes de populations dites discriminées ${ }^{4}$.

3. La législation entourant ces mesures prévoit qu'une telle application du principe de l'égalité est réputée non discriminatoire. On peut trouver le qualificatif « mesures préférentielles » à l'énoncé de ce dispositif législatif.

Le réaménagement juridique de la notion égalité, qu'a suscité la conception de ces politiques ${ }^{5}$, constitue l'un des principaux fondements de la controverse sociale qu'elles connaissent. Cette spécification du droit à l'égalité est un lieu de cristallisation des débats sur l'action positive. Elle suggère, entre autres, l'idée d'une « mise en œuvre » de l'égalité, voire de sa pratique, et éventuellement de « l'atteinte » de l'égalité. Ce sens d'une égalité «pratiquée » et que l'on peut éventuellement mesurer, occupe désormais une place importante dans la définition des enjeux égalitaires. Mais quelle place les rapports sociaux inégalitaires occupent-ils, dans la définition de ces enjeux?

\section{D'égalité et de <places socia- les> : une société en ordre}

De manière générale on estime que les politiques d'action positive constituent un nouveau mode d'attribution des places sociales, parmi lesquelles les emplois, et la notion égalité traverse l'ensemble des thèmes les plus usités dans l'appréciation sociale de ces politiques, soit le mérite, la compétence, la justice, la discrimination ou la discrimination à rebours, etc. On observe aussi, dans les appréciations savantes de ces politiques, que tant ses défenseurs que ses opposants montrent des usages de la notion égalité proches du sens commun, et que les positions même sur l'action positive, sont souvent le prolongement argumenté scientifiquement de la pensée sociale de sens commun sur ces politiques. C'est en effet ce que nous avons pu observer sur une

Les Cahiers du GRES, vol. 2, n¹, automne 2001 
période de près de cinq ans pendant lesquelles nous avons eu l'occasion d'entendre des discussions (non sollicitées de notre part) sur l'action positive dans le cadre de formations professionnelles préparées pour divers publics dans des lieux où on allait implanter un " programme d'accès à l'égalité » et dans le cadre de conversations libres entendues en divers lieux. Par exemple, la menace de la perversion de l'ordre social, que l'on définit comme égalitaire et que l'on dit assuré par le principe méritocratique (suivant la conception de l'égalité des chances la plus répandue), pourra fonder une perspective de délégitimation de l'action positive. Mais c'est aussi au nom de l'égalité, une égalité à caractère téléologique, que l'on veut concrète et pratiquée, que l'on justifie la mise en place de ces politiques. En clair, la notion égalité au centre des débats (scientifiques, mais aussi non scientifiques) sur l'action positive sert autant de justificatif d'un ordre social à maintenir que de justificatif d'un ordre social à changer.

Les débats entourant la mise en place de mesures d'action positive traduisent une conscience aiguë des interlocuteurs sur les enjeux associés à ces mesures, dont un des plus importants se rapporte à « l'ordre social » et à sa constitution, voire sa justification. Que ces politiques soient jugées subversives par certains qui y voient l'occasion de changer les modes d'attribution des places sociales ou qu'on y voit au contraire l'occasion d'une nou- velle mystification dans le fait de changer des pratiques sociales au sein d'une structure qui, elle, reste inchangée, on peut observer que c'est sur la notion d'ordre social que se fonde l'unité de ces débats ou si on veut que se rejoignent les positions <pour> et <contre> l'action positive. Être <pour $>$ ou être <contre $>$ ces politiques sociales d'action positive, c'est leur conférer un pouvoir d'impact non négligeable, qui indique la centralité de la question de l'ordre social au sein de ces débats. Toutefois, plutôt que la constitution d'un certain ordre social, la question de la constitution même des majoritaires et des minoritaires au sein de cet ordre - réputé égalitaire ou inégalitaire nous apparaît tout aussi centrale.

\section{Un point aveugle dans l'ap- préciation sociale/scientifique des politiques d'action posi- tive}

Cette question a été relativement peu exploitée au sein de la recherche sur ces politiques sociales. À ce jour, peu d'études ont procédé à l'appréciation scientifique de ces politiques sociales en notant que les groupes minoritaires (ceux auxquels sont destinés ces politiques : les femmes, les Noirs, ceux qu'au Canada on appelle les “ minorités visibles », et qu'au Québec on désigne plus volontiers par l'expression les membres des " communautés culturelles $»^{6}$, etc.) se constituent à l'intérieur de relations sociales. La catégorie minori- 
taire elle-même ne fait pas tant l'objet d'interrogations au sein de l'analyse de ces politiques. La catégorie majoritaire non plus ; les analyses se centrant plutôt sur la « gestion sociale » du fait minoritaire constaté.

Si avec l'avènement juridique de l'action positive on reconnaît que le phénomène de discrimination touche des minoritaires ou mieux, des groupes d'individus désignés socialement par le sexe, la race, l'ethnicité cette catégorisation/désignation ne fait pas elle l'objet d'examen $^{7}$. La désignation des groupes sociaux (minoritaires) en tant que processus qui s'inscrit à l'intérieur de relations sociales d'un type spécifique est peu examinée dans l'appréciation sociale des politiques dites d'action positive, sauf exception (par exemple, McAll 1990; Sociologie et sociétés 1992). Elle ne fait pas non plus l'objet d'examen dans les entreprises où on a implanté ces politiques ${ }^{8}$. Pourtant, les places sociales occupées par les majoritaires et les minoritaires sont bien au nombre des considérations sur la constitution de l'ordre social - voire sa justification -, qu'il soit pensé comme égalitaire ou comme inégalitaire. Si l'égalité au sein des discours sociaux sur l'action positive renvoie à l'idée d'ordre social, comment les majoritaires et les minoritaires sont-ils pensés au sein de cet ordre ? Quels sont alors, les rapports de ces derniers à l'égalité ? Comment ces rapports s'établissent-ils ? Ces questions permettent d'aborder la notion égalité dans une perspective relationnelle, au-delà de considérations axiologiques, soit dans une perspective proprement sociologique.

Les politiques d'action positive, leur conceptualisation et les débats scientifiques auxquels elles donnent lieu sont l'occasion de compréhension des différentes lectures qui sont faites de l'ordre constitutif des « égaux » et des « inégaux ». La conception même de l'action positive fondée à la fois sur la reconnaissance de l'inégalité d'accès, par exemple au marché du travail - sur la base des appartenances ethniques, sexuelles et raciales - et sur le principe d'une égalité actualisée, de même que les débats auxquels donne lieu la pratique de ces politiques, offrent ainsi l'occasion d'une analyse de ce que l'égalité traduit des rapports sociaux inégalitaires et ainsi des groupes majoritaires et minoritaires, que nous considérons comme se constituant au sein de ces rapports. Les fondements sociaux/politiques et la conception sociale de ces politiques, tout autant que ce qu'on en dit socialement, y compris scientifiquement, renvoient à ces relations de pouvoir et à la compréhension qu'on en a.

C'est en quelque sorte de ce point aveugle dans l'appréciation des politiques sociales d'action positive, mais aussi considérant l'absence de théorisation de la notion d'égalité en sociologie, que nous nous sommes penchées sur la structuration sociologique de la notion égalité, telle qu'on peut la

Les Cahiers du GRES, vol. 2, n¹, automne 2001 
trouver au sein des débats sur l'action positive.

C'est ainsi en regard des rapports des majoritaires et des minoritaires à l'égalité que nous parlons de structuration sociologique de la notion égalité. L'expression renvoie en quelque sorte à un postulat qui préside à notre analyse, à savoir que nous considérons que sans l'existence de rapports de pouvoir - qui constituent les majoritaires et les minoritaires - on ne saurait parler d'égalité. Entendons par là que la catégorie égalité (lorsqu'elle se rapporte à des individus ou à des situations qu'ils vivent) ne serait pas utile socialement, et donc qu'on y aurait pas recours, s'il n'y avait pas cette conscience sociale de l'existence de ces rapports sociaux de pouvoirs que ceux-ci soient reconnus ou niés, perçus ou non dans toute leur complexité, ne change rien à l'affaire. L'usage que nous faisons de l'expression la structuration sociologique de la notion égalité est aussi destiné à nommer et (à commencer) à cerner ce champ de recherche encore inexploré, où la catégorie égalité est considérée dans une perspective proprement sociologique. On pose aussi comme prémisse, on l'aura compris, que l'égalité se constitue socialement, comme idée mais aussi comme rapport (social) et comme représentation (de structure).

\section{Du point de vue méthodo- logique}

D'un point de vue méthodologique, nous avons pratiqué cette analyse sociologique de l'égalité en exploitant à des fins d'analyse discursive les catégories <majoritaire $>$ et <minoritaire> qui sont usitées en sociologie des relations ethniques et des rapports sociaux de race et de sexe et qui renvoient à l'idée de pouvoir : soit un pouvoir socialement différencié, qui fonde notamment la revendication égalitaire'. L'analyse s'est centrée sur un ensemble d'appréciations scientifiques de l'action positive de disciplines diverses (sociologie/anthropologie, sciences économiques, philosophie politique et sciences juridiques), soit l'ensemble des disciplines qui ont abordé la question de l'action positive. Ce corpus représentait des positions <pour> et $<$ contre> l'action positive et nous pouvions y trouver chacun des groupes minoritaires concernés par l'action positive. Le corpus retenu ne se voulait pas exhaustif ni comparatif mais illustratif des principales positions sur l'action positive et non sur l'égalité. Cependant, dans un corpus « pré-test », on a aussi considéré des jugements, ainsi que des documents institutionnels relatifs à l'égalité. On parle ici de textes canadiens, américains et français (pour plus de détails sur la constitution du corpus, voir Pietrantonio 1999, pp. 85-98). À cet égard, il nous faut mentionner que nous avons remarqué une certaine parité dans les textes de ces divers contextes nationaux concernant la manière d'aborder l'égalité et d'en définir les enjeux s'y rattachant. Ce qui tendrait 
à montrer la force extraordinaire de ce substrat idéologique que porte la notion d'égalité ou encore l'étendue du consensus culturel en jeu avec la pratique de l'action positive. Autre précision : les textes scientifiques de notre corpus ont été retenus en tant que représentatif du discours social sur les politiques d'action positive et non au titre de discours individuel. Par conséquent, tel qu'on le verra dans la section suivante, les références de ces textes, comme les extraits que nous produisons pour fin d'analyse, ne sont pas personnalisés. Seul un renvoi à la discipline et à la structure <pour> ou <contre> l'action positive est indiqué. II est à noter que notre analyse discursive a considéré chacun des textes dans leur entièreté et que ces derniers ont ensuite été comparés entre eux à partir d'une grille d'examen dont nous ne pouvons rendre compte ici faute d'espace. Pour l'essentiel, à travers cet examen de la production discursive scientifique sur l'action positive, qui est caractérisée par la forme d'opposition <pour $>$ /<contre $>$, nous avons cherché à reconstituer les compréhensions de la constitution de l'ordre social égalitaire ou inégalitaire en vertu desquelles les perceptions/appréciations savantes face à l'action positive sont formulées. Et cela en prenant un angle précis : soit les différentes conceptualisations scientifiques de groupes sociaux majoritaires et minoritaires par le prisme de la notion égalité. On a procédé de la sorte afin de commencer à cerner comment l'égalité est définie entre ces groupes ou comment l'égalité se définit à partir de l'appartenance à ces groupes. Nous indiquons ci-dessous quelques-uns des principaux résultats de cette étude de la structuration sociologique de la notion égalité, pratiquée à partir des catégories d'analyse sociologique <majoritaire $>$ et $<$ minoritaire $>$.

\section{La structuration sociologique de la notion égalité au sein des discours scientifiques sur l'action positive : des résul- tats d'analyse}

Concernant l'action positive, le fait suivant est à signaler : notre analyse montre que les effets des politiques sociales d'action positive sont généralement évalués non pas tant à partir de leurs incidences sur les groupes sociaux minoritaires auxquels elles sont pourtant destinées, mais bien à partir de leurs effets bénéfiques ou néfastes pour le groupe majoritaire, le groupe social de référence ${ }^{10}$. Les effets positifs comme les effets négatifs de ces politiques concernent d'abord la structure sociale. L'extrait que nous présentons ci-dessous est issu d'un texte de philosophie politique à structure <pour> l'action positive. II s'agit des derniers énoncés d'un texte d'une vingtaine de pages destiné à faire valoir l'utilité de la mise en œuvre des politiques d'action positive. Signalons que les premiers énoncés du texte annonçaient le développement "d'une théorie adéquate de l'égalité » parce que « [l]es difficultés théoriques posées

Les Cahiers du GRES, vol. 2, n¹, automne 2001 
par la notion d'action positive tiennent en fin de compte à l'ambiguïté $d u$ concept de l'égalité » (nos italiques). Sans développer plus avant cette idée d'ambiguïté de l'égalité, l'argumentaire du texte se déploiera en fait par l'exemplification d'un " exercice de réciprocité des perspectives » pratiqué au titre de « principe de la justice en tant que réversibilité » dont la finalité consiste à ... « assurer le maintien de la probité du système »:

« [...] Un Blanc intéressé à préserver la structure fondamentale de la société, et à réaliser l'intégration de tous les membres de la société en un système uni de production et de distribution, serait par conséquent d'accord pour implanter des programmes provisoires d'action positive même s'ils ont pour effet de diminuer ses propres chances d'obtenir certaines denrées rares. En somme, vue à partir de la réciprocité totale de toutes les perspectives pertinentes, l'action positive ne prive finalement aucun individu de son droit d'être traité en égal ». [...] En outre, l'action positive [...] remanie les places de certains individus, mais par là même elle maintient intacte les structures $d u$ pouvoir et les hiérarchies professionnelles existantes » (nos italiques).

C'est à la légitimation de l'ordre social qu'est orienté le déploiement argumentatif de ce texte, tout autant qu'à la légitimation de l'action positive - pour le groupe social de référence (« un Blanc ») auquel est en fait destiné ce texte. La légitimité de l'action positive pour les minoritaires n'est ici pas questionnée. Elle ne constitue pas l'objet de ce texte, non plus que le phénomène de discrimination. On constate aussi qu'il y a des <places $>$ (sociales) et que ces " places de certains individus" sont en fait celles du groupe social de référence (celui « intéressé à préserver la structure fondamentale de la société, et à réaliser l'intégration de tous... »), soit : le sujet social ou l'agent; celui qui agit («<préserve> la structure »>; <est ... « d'accord pour »>; <laisse sa « place »>; < réalise « l'intégration de tous " >). Mais attirons l'attention sur cet autre extrait : « ...l'action positive ne prive finalement aucun individu de son droit d'être traité en égal ». Dans ce texte social, nous n'avons rencontré aucune occurrence où l'action positive aura été examinée sous cet angle précis, soit $<$ priver un individu de son droit d'être traité en égal>, pour les groupes sociaux minoritaires. Les " individus » de l'expression "ne prive finalement aucun individu... » ne sont en fait que des individus du groupe social de référence, le groupe majoritaire. Nulle part en effet, dans ce texte social à structure <pour> ni d'ailleurs dans aucun texte social sur l'action positive, lu ou entendu, scientifique ou de sens commun, n'avons-nous rencontré ce type d'énoncé pour la catégorie sociologique <minoritaire>. Dans 
l'ensemble de notre corpus, les seules occurrences où l'action positive est dite spriver un individu de son droit d'être traité en égal > sont les occurrences qui concernent le groupe sociologique <majoritaire $>$. La conclusion de ce texte paraît concerner <tous les individus> alors qu'en fait elle ne concerne que les individus du groupe social de référence - le seul sujet social possible dans les occurrences où l'action positive est associée à une privation, quelle qu'elle soit et cela à partir d'un exercice dit de “ réciprocité » (« la justice en tant que réversibilité ») qui suppose une symétrie sociale, ici en regard de l'égalité, des groupes sociaux en examen.

De même, la dynamique du fonctionnement de cette structure sociale est décrite en regard d'actions posées par le groupe majoritaire; actions qui rendent compte ainsi d'une pratique de l'égalité dont on défend l'existence. En ce cas, on se réfère à l'idée de libre circulation du marché et des biens et à l'idée de compétition.

« [...] la consécration légale ou institutionnelle du pouvoir d'échanger entre deux agents aux conditions qui élèvent forcément le bien-être de l'un et de l'autre des deux échangeurs » (nos italiques).

L'extrait est relevé d'un texte de sciences économiques, dont le déploiement discursif est destiné à prévenir contre la menace à la " liberté » que constitue la pratique de l'action positive. Dans l'enchaînement discursif, l'égalité des chances, qui est « tradition occidentale ", sert un ordre marqué par l'efficacité et motivé par la « liberté ». Le pouvoir des «échangeurs» assure la dynamique d'un marché aux effets bénéfiques... sur ces derniers. Dans ce texte à structure <contre> l'action positive, les femmes ne sont en aucune occurrence considérées comme des « échangeurs ».

« En l'absence de discrimination, tant que les femmes auront des enfants et qu'elles auront une perception semblable de leur carrière, le marché l'enregistrera et créera des écarts de traitements. [...] Si les femmes évoluent, le résultat s'enregistrera quelque part dans le marché » (nos italiques).

"L'entreprise qui ferait la bêtise de discriminer aurait des coûts de production plus élevés, ses profits seraient plus bas ». [Discriminer] «pénaliserait l'entreprise : Voilà la position-clé. C'est l'employeur qui souffrirait de discrimination » (nos italiques).

Tel que l'indiquent ces deux extraits, le cœur de ce texte de sciences économiques est motivé par la (re)définition de la « discrimination ". Le texte analyse ainsi le phénomène de discrimination systémique que vivraient «les femmes » en faisant valoir l'idée que ce

Les Cahiers du GRES, vol. 2, n¹, automne 2001 
phénomène serait le résultat non pas du «marché » ou d'une “ entreprise » mais plutôt d'une nature spécifique des femmes, exprimée par l'entremise de la maternité (le fait <d'avoir> des enfants) et ses effets psychologiques sur la «perception... ». Les femmes sont ici, par trait de nature, hors du champ de « l'égalité des chances » par leur propre "perception », et devraient <évoluer> afin de rejoindre ce champ des «échangeurs »-dont on constate qu'eux et eux seuls entretiennent un rapport de contiguïté avec ce champ, par leur seule médiation personnelle : l'action (<l'échange>) plutôt que la «perception ».

L'égalité dans les discours sociaux scientifiques sur l'action positive, dont l'actualisation est largement débattue, n'est cependant pas remise en cause. En fait, elle se réfère principalement à une situation sociale générique, soit l'absence de discrimination ou d'entraves. Dans l'ensemble des textes, l'égalité veut dire <ne pas être de ceux qui sont discriminés>, soit être dans ce lieu social de l'égalité, le plus souvent défini, en implicite, par le marché du travail. Elle veut dire aussi ne pas être « défavorisé » et sert à " se faire valoir ", sens qui indique l'idée d'une reconnaissance sociale que favoriserait « l'accès (sans entrave) à l'égalité ». En ce sens, notre analyse montre une égalité qui se définit essentiellement par les conditions sociales qu'on peut dire être le fait de majoritaires, par ailleurs idéalisées par tous, majoritaires et minoritaires.

« [les minoritaires]... vantent peu les mérites de l'action positive, sauf pour souligner qu'elle donnera peut-être une pulsion aux immigrants pour se faire valoir, une fois qu'ils seront mis sur un même pied d'égalité » (nos italiques) (texte de sociologie/anthropologie à structure <contre> l'action positive).

Notons, au passage, la référence implicite (soit, non spécifiée) du « même » dans l'expression "le même pied d'égalité ». Ainsi que nous l'avons signalé plus haut, les deux thématisations de l'égalité les plus importantes que nous avons relevées dans l'ensemble de notre corpus sont l'égalité revendiquée (l'égalité à atteindre) et l'égalité défense d'un état (l'égalité à préserver). Mais l'égalité apparaît aussi comme un <état social> pour la catégorie de référence (majoritaire) d'abord et avant toute analyse, ce qui revient à dire que la situation sociale de référence, celle des majoritaires, que traduit la notion égalité (par exemple dans son sens le plus prégnant : le fait de « n'être pas discriminé » ou “ défavorisé »), ne fait pas l'objet d'analyse. Dans aucun des textes analysés, l'égalité n'est <état social> pour la catégorie minoritaire d'abord et avant toute analyse. Ce fait que notre pratique d'analyse a pu mettre au jour révèle un défaut de symétrie de conceptualisation du rapport des 
uns et des autres majoritaires et minoritaires à l'éga-lité ${ }^{11}$. II y a bel et bien une dualité de l'égalité, que l'on peut identifier au sein de textes sur l'action positive - et que l'on peut relever par exemple par l'expression ironique " certains sont plus égaux que d'autres $\gg^{12}-$, mais cette dualité n'est pas symétrique, et ne paraît pas non plus relationnelle. Autrement dit, on ne considère nulle part, dans aucune occurrence donc, que l'égalité s'établit à partir de rapports so-ciaux - que ceux-ci soient de groupe (s) à groupe (s) ou d'individu (s) à individu (s) -, mais dans tous les textes examinés on retrouve une égalité qui s'établit dans le rapport des individus à la structure sociale.

Notre pratique d'analyse nous a pourtant placé devant le constat suivant: l'égalité à laquelle « accèdent » les minoritaires n'est pas une égalité entre minoritaires mais une égalité dont le référent est le groupe social majoritaire, tant dans sa dimension concrète que symbolique. L'égalité, on peut le comprendre alors, n'a de sens que dans une perspective relationnelle, même si ce fait n'apparaît dans aucun des textes étudiés; que cette relation se fasse aussi intra-groupe n'apparaît pas non plus de manière explicite. On peut cependant la lire de manière implicite dans l'expression les <libres échangeurs> - qui feraient $<$ fonctionner un ordre social de gens libres... d'entrer en compétition>. Dans les textes scientifiques sur l'action positive, c'est ainsi la dynamisation de l'égalité qui pose problème, et non pas l'égalité elle-même. Mais on doit ajouter : la dynamisation de l'égalité pour les minoritaires.

Ainsi, l'égalité existe. Et elle est à atteindre ou à protéger. On peut en trouver une sorte de preuve dans l'idée suivante observée au sein de notre corpus : on ne songe pas à débattre longuement de l'action positive pratiquée par des minoritaires, qui pratiqueraient pour ainsi dire une « embauche préférentielle » en s'embauchant entre eux et en excluant les majoritaires ${ }^{13}$. L'extrait que nous présentons ci-dessous est tiré d'un texte en sociologie/anthropologie à structure <contre> l'action positive :

« ... il y avait quasi unanimité chez nos interlocuteurs à ne pas mettre le phénomène de sous-économie ethnique (italiques du texte) - des entreprises créées par des minoritaires et qui emploient de préférence des travailleurs de même origine - sur le même pied que l'exclusion de minoritaires par des majoritaires. Presque tous approuvent ces initiatives, dans la mesure où il n'y a pas d'exploitation des immigrants, même s'ils regrettent l'existence de ghettos d'emplois. Plutôt que de considérer ces pratiques contradictoires avec l'esprit de l'action positive, il s'agit de moyens d'accès non pas à l'égalité, mais à l'emploi pour ceux qui n'en auraient pas

Les Cahiers du GRES, vol. 2, n¹, automne 2001 
autrement... » (nos italiques).

Si on déplore ce genre de pratique d'embauche «préférentielle », chez les minoritaires par des minoritaires, en disant qu'elle crée des " ghettos d'emploi », on ne songe pas apparemment à débattre de ce type d'action positive avec le thème de l'égalité (« [p]lutôt que de considérer ces pratiques contradictoires avec l'esprit de l'action positive, il s'agit de moyens d'accès non pas à l'égalité, mais.... »). On sait alors que l'égalité ne se trouve pas au sein de ce type d'emplois chez les minoritaires. "L'embauche préférentielle » chez les minoritaires ne porte pas atteinte à l'égalité, comme on conçoit que ce soit le cas chez les majoritaires. Le thème de l'égalité pour débattre des politiques d'action positive (chez les majoritaires) apparaît ici clairement - dans une échappée de sens. Notons au passage qu'on ne songe pas non plus au thème du " mérite » pour qualifier les motifs d'attribution de ces emplois. Ce fait montre l'importance de l'idéologie méritocratique dans la conception de l'égalité, ou sa représentation, et la centralité de la notion <égalité> comme descriptive du lieu social $<$ majoritaire>, lequel est circonscrit principalement par «le marché de l'emploi » - mais un marché de l'emploi qui n'est pas qualifié de " ghetto d'emploi ». On constate ainsi que l'expression " ghetto d'emploi » ne renvoie pas tant au type d'emploi exercé mais bien aux groupes d'individus qui le pratiquent ${ }^{14}$.
Enfin, suivant notre pratique d'analyse, on peut dire que dans toutes les occurrences et thématisations diversifiées de l'égalité, observées au sein de textes scientifiques sur l'action positive, il est un référent qui oriente sa définition sociale, mais qui ne quitte jamais le lieu de l'implicite. En vertu de ce référent on parlera alors de « déviation » de l'égalité ou de " dérogation » au principe de l'égalité qu'instaurerait la pratique de l'action positive, et cela que l'on soit <pour> ou $<$ contre $>$ les pratiques d'action positive.

"L'objection majeure soulevée contre les programmes d'action positive vient $d u$ fait qu'ils procurent un traitement préférentiel aux minorités, au détriment du groupe majoritaire, et cela constitue de la discrimination à rebours. N'estil pas paradoxal, en effet, que dans le but d'en arriver à une plus grande égalité, à long terme, l'on doive prendre des mesures qui, à court terme, vont directement à l'encontre du principe même de l'égalité »(nos italiques).

Dans cet extrait issu d'un texte de sciences juridiques à structure <pour> l'action positive, observons seulement que, par un procédé discursif de contiguïté, faire " au détriment du groupe majoritaire » (ici par un « traitement préférentiel aux minorités »), c'est <aller «à l'encontre du principe même de l'éga- 
lité »>. Dans un autre extrait du même texte, on peut voir le référent implicite de l'égalité spécifié :

« Si la majeure partie du problème de l'égalité pouvait se résoudre en démontrant que les femmes ont les mêmes capacités que les hommes... » (nos italiques).

On peut lire ici le référent qui oriente le sens social de l'égalité mais aussi le référent des " capacités » sociales - qui mènent à l'égalité. Ce référent qui oriente la conduite du discours social sur l'égalité et l'asymétrie de sa conceptualisation selon les catégories majoritaires et minoritaires ne paraît pas porté à la conscience des producteurs de ces textes sociaux. Cette obstruction perceptive découle, nous semble-t-il, d'une évidence sociale partagée par tous, majoritaires et minoritaires, et non questionnée, à savoir que l'égalité est pensée comme un symétrique social. Derrière cette apparente symétrie, un sort différent est pourtant fait à l'égalité des uns et des autres avant toute analyse et à l'analyse. Au sein de notre corps de textes, c'est le groupe majoritaire en tant que groupe matériel autant que groupe symbolique à valeur de référence que nous avons rencontré le plus souvent. Suivant les chaînes de sens où nous retrouvions ce majoritaire, on a pu observer de manière constante que le rapport de ce dernier à l'égalité est établi par contiguïté et non par opposition comme c'est le cas pour la catégorie
$<$ minoritaire >. À titre d'exemple ici, mentionnons que le thème de “ l'égalité dans la différence 》 (aussi présent dans les textes scientifiques sur l'action positive) ne concerne que les minoritaires - les seuls à être définis (socialement) par la notion « différence », que l'on oppose à « égalité $»^{15}$. Dans un autre extrait du texte de philosophie politique à structure <pour $>$, on peut lire ci-dessous un exemple de dissymétrie de conceptualisation des majoritaires et des minoritaires devant l'égalité mais aussi devant l'appréciation d'une situation sociale qui peut être commune, soit <postuler pour un emplois:

« [...] En effet, à partir de la réciprocité des perspectives, le désir d'action positive afin d'effectuer la transition entre l'infériorité et l'égalité semble être moralement supérieur à la préoccupation du Blanc de réduire l'angoisse causée par la peur d'échouer pour l'emploi qu'il postule » (nos italiques).

On peut observer que « l'égalité » et « l'infériorité » sont deux espaces sociaux séparés; qu'on ne peut être (sociologiquement) dans l'un (l'espace social de l'égalité) et dans l'autre (l'espace social de l'infériorité) à la fois, puisqu'il y a « transition à effectuer » (pour les minoritaires seulement). Notons aussi que l'opposé social de l'égalité n'est pas ici l'inégalité ou les inégalités mais bien « l'infériorité » laquelle infériorité ne connote pas exclusivement des référents de

Les Cahiers du GRES, vol. 2, n¹, automne 2001 
socialité et exclut certainement toute analyse en termes de rapports sociaux. De même, on remarquera que "l'angoisse causée par la peur d'échouer pour l'emploi qu'il postule » est restreinte dans son usage au groupe social de référence (ici le «Blanc »). Nulle part dans ce texte ni au sein d'autres textes à structure <contre> et à structure <pour> n'avons-nous rencontré d'occurrence où " l'angoisse causée par la peur d'échouer pour l'emploi qu'il postule » est le fait des minoritaires. Non pas que ce ne soit pas possible, mais le fait n'est pas considéré. "La peur d'échouer pour l'emploi qu'il [le Blanc] postule » n'est causée ici ni par une « infériorité " ressentie ou désignée par d'autres ni par crainte de compétence non reconnue mais bien par la pratique de l'action positive. Dans ce même sens, la forme <postuler pour un emploi> est généralement réservée au sein de ce texte au groupe social de référence, alors que l'on trouve la forme < « convoiter " un emploi> pour la catégorie minoritaire. Connotation : convoiter... « le bien d'autrui » ou " désirer une chose qui appartient à un autre »(Le Petit Robert 2000).

\section{Remarques conclusives}

L'une des conclusions importantes de notre analyse 'est que l'égalité, telle qu'elle est usitée dans les textes scientifiques sur l'action positive, ne remet pas en cause l'ordre social existant. S'il y a revendication égalitaire, celle-ci n'émane plus seulement de groupes minoritaires et ces derniers réclament, pour ainsi dire rapidement, surtout des aménagements. Avec l'action positive, la lutte des minoritaires pour « l'atteinte de l'égalité » se fait au sein d'une structure déjà existante, et dont on prétend qu'elle peut être « adaptée ». C'est là que se situe principalement la discussion sur les enjeux égalitaires. Contrairement au projet sociopolitique du $18 \mathrm{e}$ siècle, ce n'est pas la structure sociale qu'il faut changer, mais bien les mesures d'accès pour les groupes sociaux minoritaires. Cette distinction majeure éclaire peut-être implicitement un des sens sociaux que recouvre la notion égalité tel qu'on a pu l'observer au sein de textes scientifiques <pour> et <contre> l'action positive : soit d'être descriptive de la structure sociale et du groupe social de référence. Notre analyse montre que ces politiques d'action positive sont aussi pensées dans une perspective de maintien d'une structure sociale où le pouvoir est différencié par l'appartenance à des catégories (minoritaires) de sexe, de race, d'ethnicité. Les types ou systèmes de compréhension des sources des inégalités et de l'égalité que révèlent la controverse sur l'égalité et la justice au sein des débats sur l'action positive achoppent sur la saisie de la spécificité des rapports de pouvoir entre majoritaires et minoritaires. Entre les positions qui indiquent la nécessité de combattre la discrimination par ces politiques et celles qui indiquent que ces politiques créent de la discrimination envers 
les majoritaires et pervertissent le système, la catégorisation/désignation/spécification des minoritaires demeure impensée et avec elle, les rapports sociaux de pouvoir qui les constituent tels, catégorisés, désignés, spécifiés, jusqu'à l'intérieur de la norme symbolique et concrète que constitue l'égalité. À cet égard, le type de compréhension des rapports entre les majoritaires et les minoritaires demeure capital, et le rapport des majoritaires et des minoritaires à l'égalité à être documenté plus avant - dans une perspective sociologique.

Notre étude révèle que ce qui est disputé avec la notion égalité, c'est entre autres un lieu social, une situation sociale qui sont exempts d'entraves ou de discrimination. Ainsi, on s'aperçoit que l'égalité au sein des textes sur l'action positive décrit non seulement une structure sociale, perçue comme égalitaire, mais tout autant une situation sociale idéalisée et pour telle revendiquée, qu'une situation sociale existante, celle du majoritaire qui ne subit pas de discrimination - sur le marché de l'emploi. II y a là bien peu d'ambiguïté.

\section{Notes}

1 Ce que nous appelons des «politiques d'action positive » connaît diverses appellations, dont la plus connue est sans doute l'appellation américaine " Affirmative Action Program ». Au Québec, on parle de «pro- grammes d'accès à l'égalité ", alors que les autres provinces canadiennes usent de l'appellation «Équité en emploi ». En France, on a utilisé l'expression " programmes d'égalité professionnelle », mais aussi « discrimination positive »- pour ne nommer que ces quelques sociétés. Nous retenons l'expression « action positive » comme une appellation générique.

2 Pour une analyse du phénomène de discrimination systémique au Québec voir : Chicha-Pontbriand, MarieThérèse (1989); Garon, Muriel (1986); Dusseault, Ginette (1983); McAll, Christopher (1990).

3 Les mesures d'action positive sont régies par des conditions que l'on retrouve dans les conventions internationales contre la discrimination sexuelle et raciale. À ce titre, mentionnons la Convention internationale sur l'élimination de toutes les formes de discrimination à l'égard des femmes de l'Organisation des Nations Unies et la Convention internationale sur l'élimination de toutes les formes de discrimination raciale.

4 Cette position a été défendue notamment par la Juge Rosalie Silberman Abella (1984) ainsi que par la Commission des droits de la personne et des droits de la jeunesse du Québec.

Les Cahiers du GRES, vol. 2, n¹, automne 2001 
5 Au Canada, la Charte canadienne des droits et libertés élaborée autour du droit à l'éga-lité fût amendée afin de prescrire un traitement égalitaire à partir de l'appartenance de catégories raciales, ethniques et sexuelles, ainsi qu'à partir d'autres catégories liées à un processus de minorisation tels le handicap physique ou mental. II en fut de même au Québec.

6 L'expression est encore usitée malgré la loi constitutive du Ministère des Relations avec les citoyens et de I'Immigration (MRCI), qui a remplacé le Ministère des Communautés culturelles et de l'Immigration (MCCI), en 1996 (nos italiques).

7 On peut toutefois s'inquiéter du caractère factice de certaines catégories, comme on l'a fait notamment au Canada avec la catégorie « minorité visible », ou encore dénoncer les effets de l'usage de ces catégories par l'État en invoquant « l'ethnicisation » et ses manquements supposés à l'universalisme, tel qu'en font foi les débats actuels sur le pluralisme normatif, mais rarement aborde-t-on ces catégories (majoritaire et minoritaire) comme produites à l'intérieur d'un rapport social.

8 Notons que cette dimension de la recherche sur l'action positive, soit la recherche empirique dans les entreprises qui ont mis en place des mesures d'action positive, est restée relativement peu développée en comparaison des travaux qui se sont penchés sur les effets macro-sociaux de ces politiques.

9 Le développement de notre pratique d'analyse s'est appuyé sur une approche théorique qui considère les groupes sociaux majoritaires et minoritaires dans leurs dimensions constitutives. Voir notamment Guillaumin (1972); Juteau (1999); Pietrantonio (2000); Pietrantonio (1999).

10 Les exceptions à cet égard sont le fait de travaux qui se penchent plus spécifiquement sur le phénomène de discrimination systémique pour l'appréciation scientifique de ces politiques.

11 Nous avons observé ailleurs (Pietrantonio 2000) cette symétrie déficiente dans la saisie scientifique des majoritaires et des minoritaires, au sein de problématisations sociales diverses qui les mettent en scène. Cette symétrie déficiente n'est ainsi pas le seul fait de la notion égalité - le fait est à signaler.

12 Expression présente dans notre corpus et rendue célèbre par la fable de George Orwell (1981) [1945], La ferme des animaux : "Tous les animaux sont égaux, mais (il semble que cela ait été rajouté) il y en a qui le sont plus que d'autres ».

13 Pensons notamment à l'industrie du taxi à Montréal où un groupe 
de propriétaires Haïtiens embauche des chauffeurs Haïtiens.

14 Pour reprendre précisément notre exemple de l'industrie du taxi, il ne viendrait à l'esprit de personne de qualifier de " ghetto d'emploi » une «flotte de taxi » (comme on dit dans le milieu) où les propriétaires et conducteurs sont des «Blancs ».

15 Cette thématisation de « l'égalité dans la différence » est problématique à maints égards, notamment parce que ces deux catégories «égalité » et « différence » sont souvent usitées comme des donnés. Les relations de pouvoir qui sont constitutives de l'idée de différence sont alors oblitérées - sans parler des rapports constitutifs de l'égalité, dont on aura compris qu'ils sont pour nous un objet d'analyse. Une telle thématisation de "l'égalité dans la différence » n'est pas récente. On en trouve des traces au $18 \mathrm{e}$ siècle lors des débats sur la place des femmes et leur rapport à l'égalité, que l'on est à constituer alors en principe juridique. Voir à ce sujet Capitan, Colette (1993), La nature à l'ordre du jour. 17891793, Éditions Kimé, Paris; Varikas, Eleni (1987), « Droit naturel, nature féminine et égalité des sexes ", L'Homme et la société, nos 85-86, pp. 98-111, 2e semestre.

\section{BIBLIOGRAPHIE}

Capitan, Colette, 1996. « Nation, Nature et statut des personnes au cours de la Révolution française ", Mots. Les langages du politique, no 49, pp. 18-28.

Capitan, Colette, 1993. La Nature à l'ordre du jour. 1789-1793, Éditions Kimé, Paris, 179 p.

Chicha-Pontbriand, Marie-Thérèse, 1989. Discrimination systémique. Fondement et méthodologie des programmes d'accès à l'égalité en emploi, Les Éditions Yvon Blais inc., Cowansville (Québec), $197 \mathrm{p}$.

Commission des droits de la personne et des droits de la jeunesse du Québec, 1998. Les programmes d'accès à l'égalité au Québec. Bilan et perspectives. Maintenir les acquis. Élargir le champ d'action. Résumé, Montréal, Gouvernement du Québec.

Commission des droits de la personne, 1988. L'accès à l'égalité dans l'emploi. Guide d'élaboration d'un programme volontaire, Gouvernement du Québec.

Delphy, Christine, 1995 . «Égalité, équivalence et équité : la position de l'État français au regard du droit international ", Nouvelles questions féministes, vol. 16 , no 1 , pp. 5-58.

Dusseault, Ginette, 1983. La discrimination sur le marché du travail : le cas des employés de

Les Cahiers du GRES, vol. 2, n¹, automne 2001 
bureau, Thèse de doctorat, présentée à l'Université McGill, Québec.

Garon, Muriel, 1986. «Pour une approche intégrée de la discrimination systémique : convergences et contributions des sciences sociales et du droit ", Revue de droit de l'Université de Sherbrooke, no 17, pp. 191-242.

Guillaumin, Colette, 1972. L'idéologie raciste. Genèse et langage actuel, Paris, Mouton, $247 \mathrm{p}$.

Juge Abella, R.S., 1984. Égalité en matière d'emploi. Rapport d'une commission Royale, ministère des Approvisionnements et Services, Canada.

Juteau, Danielle, 1999. L'ethnicité et ses frontières, Montréal, Les Presses de l'Université de Montréal, $226 \mathrm{p}$.

McAll, Christopher, 1990. « Discrimination et action positive : de l'irrationalité culturelle à la rationalité collective », Ida SimonBarouh et Pierre-Jean Simon (dir.), Les étrangers dans la ville. Le regard des sciences sociales, L'Harmattan, pp. 266-276.

Orwell, George, 1981 [1945]. La ferme des animaux : fable, Paris, Éditions Champ libre, $151 \mathrm{p}$.

Pêcheux, Michel, 1979. « Un exemple d'ambiguïté idéologique : le rapport Mansholt ", Technologies, idéologies et pratiques,
Publications de l'Université de Provence, Aix, avril-juin, pp. 4-83.

Pietrantonio, Linda, 2000. «Une dissymétrie sociale : rapports sociaux majoritaire/minoritaires », Bastidiana, no. 29-30, janvier-juin, pp. 151-176.

Pietrantonio, Linda, 1999. La construction sociale de la (dé)légitimation de l'action positive ou l'envers de l'égalité, Thèse de doctorat présentée au département de sociologie de l'Université de Montréal, Québec, 288 p.

Sociologie et sociétés, 1992. vol. 24, no 2, Les Presses de l'Université de Montréal.

Varikas, Eleni, 1987. « Droit naturel, nature féminine et égalité des sexes ", L'Homme et la société, no 85-86, pp. 98-111. 